\title{
EDITORIAL
}

\section{The Quest for Quality: Does Network Participation Matter?}

\author{
Herminia Palacio, MD, MPH \\ Harris County Public Health and Environmental Services, Houston, TX 77027, USA.
}

$\mathrm{J}$ Gen Intern Med 22(10): 1482

DOI 10.1007/s11606-007-0327-9

(c) Society of General Internal Medicine 2007

$\mathrm{T}$ he clarion call to focus on quality of health care has been heeded by many and has led to a body of research aimed at identifying factors associated with improved quality of care. ${ }^{1-6}$ In this issue, Friedberg et al. ${ }^{7}$ explore the association between quality of care-as measured by Health Plan Employer Data and Information Set (HEDIS) indicators-and two characteristics of physician group practices. Specifically, Friedberg et al. ${ }^{7}$ examined whether HEDIS measures varied by the size of the physician group and by whether or not the physician group was a member of a "physician network". They reviewed data from five managed care organizations that encompassed contracts with more than 4,300 primary care physicians in group practices in Massachusetts. Friedberg et al. ${ }^{7}$ found that in multivariate models, physician group size was associated with only 3 of 12 HEDIS measures. Of note, whereas for two diabetes-related HEDIS measures mediumsized groups had more favorable performance scores, for another diabetes-related HEDIS measure, small physician groups had the more favorable performance scores.

The data regarding an association between network affiliation and HEDIS performance yielded more consistent results. Specifically, Freidberg et al. found that in multivariate analyses, network affiliation was associated with higher HEDIS performance scores in 8 (of 12) measures. These findings were fairly robust, overall holding up well under various conditions such as excluding the two highest performing networks from one analysis and excluding the two largest networks from another analysis.

How does the examination of these organizational constructs (group size and network affiliation) contribute to our understanding of quality care and what are the policy implications of the data presented by the findings by Friedberg et al.? ${ }^{7}$ From a policy perspective, it is particularly important to consider interventions that are not just effective but are also feasible, efficient, relatively generalizable to a variety of healthcare settings. Furthermore, interventions that can be applied at the system level are attractive, as they do not rely solely on the vagaries of individual physician behavior.

The analyses on group size failed to reveal a pattern that could be used to develop and test an intervention that has high potential for meeting even the fundamental policy criteria of being effective. Why? Because in their analysis, there was no preponderance of evidence that any one group size had a distinct advantage over another vis-à-vis the selected HEDIS performance measures. This is not necessarily a disappointing

Published online July 31, 2007 result. Indeed, as the ability of primary care physicians to organize themselves into medium or large group practices is likely constrained by a number of factors (geographic location, population density, etc.), the data by Friedberg et al. ${ }^{7}$ offer some comforting assurance that there are not huge missed opportunities to improve quality of care imposed by the constraints on altering group practice size.

The analyses on network affiliation yielded more promising policy implications. Network affiliation was associated with improved HEDIS performance scores across a majority of measures. The policy implications are intriguing because if network affiliation is truly an important player in the causal pathway toward quality, then developing broad-based strategies to enhance physician group enrollment into networks would be feasible relatively efficiently and potentially generalizable to a variety of practice settings. Indeed, during the 1990s, there was rapid expansion of the number of physicians affiliated with networks. ${ }^{8}$ At the end of the day, however, the findings presented by Friedberg et al. ${ }^{7}$ are intriguing and provocative, but insufficient in and of themselves to be the sole impetus for sound policy development. Their findings, however, when taken into account with studies looking at the impact of other additional organizational strategies such as pay for performance $^{9}$ should provide healthcare markets with sufficient information to begin to implement and test the impact of specific interventions on quality measures in a prospective manner.

Corresponding Author: Herminia Palacio, MD, MPH; Harris County Public Health and Environmental Services, 2223 West Loop South, Houston, TX 77027, USA (e-mail: hpalacio@hcphes.org).

\section{REFERENCES}

1. Baker LC, Hopkins D, Dixon R, Rideout J, Geppert J. Do health plans influence quality of care? Int J Qual Health Care. 2004;16:19-30.

2. Dean Beaulieu N, Epstein AM. National Committee on Quality Assurance health-plan accreditation: predictors, correlates of performance, and market impact. Med Care. 2002;40:325-37.

3. Fleming B, Silver A, Ocepek-Welikson K, Keller D. The relationship between organizational systems and clinical quality in diabetes care. Am J Manag Care. 2004;10:934-44.

4. Gillies RR, Chenok KE, Shortell SM, Pawlson G, Wimbush JJ. The impact of health plan delivery system organization on clinical quality and patient satisfaction. Health Serv Res. 2006;41:1181-99.

5. Himmelstein DU, Woolhandler S, Hellander I, Wolfe SM. Quality of care in investor-owned vs not-for-profit HMOs. JAMA. 1999;282:159-63.

6. Committee on Quality of Health Care in America IOM. To Err is Human: Building a Safer Health System. Washington, DC: National Academy Press; 1996.

7. Friedberg MW, Coltin KL, Pearson SD, et al. Does affiliation of physician groups with one another produce higher quality care? J Gen Intern Med. 2007;22.

8. Robinson JC. Consolidation of medical groups into physician practice management organizations. JAMA. 1998:279:144-9.

9. Mehrotra A, Pearson SD, Coltin KL, et al. The response of physician groups to P4P incentives. Am J Manag Care. 2007;13:249-55. 